\title{
RESISTÊNCIA INTRÍNSECA DA FIBRA DE ALGODÃO DETERMINADA ATRAVÉS DE CORREÇÃO DO ÍNDICE PRESSLEY $\left({ }^{1}\right)$
}

\author{
IMRE LAJOS GRIDI-PAPP $\left({ }^{2}, 4\right)$, JULIO ISAO KONDO $\left({ }^{3}, 4\right)$, NELSON PAULIERI \\ SABINO $\left({ }^{3},{ }^{4}\right)$ e MILTON GERALDO FUZATTO $\left({ }^{2}\right)$
}

\begin{abstract}
RESUMO
E proposta correção do índice Pressley 1/8" no sentido de se obterem dados em termos de resistência média por fibra individual, que foi chamada resistência intrínseca (RI), e demonstrou-se que ela pode ser estimada pela fórmula: $R I=15.0,11383\left(P / p_{2}\right)\left(p_{1} / L \cdot L_{2,5 \%}\right)$, onde $P$ é a leitura no aparelho Pressley, $\mathrm{p}_{2}$ o peso da mecha de fibras, $L, L_{2,5 \%}$ e $p$ são, respectivamente, a leitura inicial, o comprimento $2,5 \%$ e o peso de fibras no pente do fibrógrafo. Estudos realizados pelos AA. no Laboratório de Fibras do IAC, comparando as duas medidas de resistência na ausência de variaçōes genéticas, mostraram que, enquanto o f́ndice Pressley não se correlacionou com maturidade e índice Micronaire, a resistência intrínseca apresentou coeficientes de correlação altamente significativos com ambos os caracteres. Considerando-se os principais caracteres tecnológicos da fibra, a maturidade foi o caráter que mais contribuiu para as variaçoes da resistência intrínseca em análise da regressão múltipla. Em estudo de 22 ensaios regionais de variedades, essa medida de resistência proporcionou maior discriminação que o índice Pressley $1 / 8^{\prime \prime}$.
\end{abstract}

Termos de indexação: algodão; resistência intrínseca; Pressley corrigido.

(1) Recebido para publicação em 11 de junho de 1984.

(2) Seção de Algodão, Institu to Agronômico (I AC), Caixa Postal 28, 13100 Campinas (SP).

( $\left.{ }^{3}\right)$ Seção de Tecnologia de Fibras, IAC.

$\left({ }^{4}\right)$ Com bolsa de suplementaçāo do CNPq. 


\section{INTRODUÇÃO}

A resistência à tração é uma das características físicas mais importantes da fibra de algodão: afeta diretamente a tenacidade do fio e, indiretamente, a qualidade dos tecidos. $\mathrm{E}$ afetada decisivamente pelo grau de maturidade do algodão produzido e, logo, por todos os fatores que influem no processo de maturação, pois estes podem favorecer ou desfavorecer a deposição de celulose nas fibras, conferindo-thes maior ou menor resistência. Há outras características da fibra que influem em sua resistência, como o estado de polimerização da celulose, o ângulo que os feixes de fibrilas formam com o eixo da fibra e o número de pontos de inversão da orientação desses feixes (DE COENE \& VERSCHRAEGE, 1963). O aspecto da maturidade em termos de grossura da parede da fibra assume, porém, destacada importância, em vista do problema específico que introduz nas medições usuais da resistência em uma mecha de fibras.

A medição do grau médio de resistência de uma amostra de fibras de algodão tem apresentado sérias dificuldades. Medições sobre fibras individuais requerem aparelhos ou procedimentos refinados, são morosas e exigem a solução do complexo problema de amostragem (DELUCA, 1964). Na realidade, determinações sobre mechas de fibras paralelas são mais práticas e rápidas, e levaram ao uso de aparelhos como o de CHANDLER (1926), de Clemson (BURLEY JR. \& CARPENTER, 1955, PRESSLEY, 1942), e o stelômetro (BURLEY JR. \& CARPENTER, 1955). Todos eles implicam a correção do resultado para um mesmo peso de mecha ou amostra. O problema acima mencionado surge pela observação durante o uso do aparelho "Pressley" nos trabalhos de melhoramento genético, de que uma mesma linhagem ou variedade tende a apresentar valores de resistência maiores quando piora ligeiramente o grau de maturidade das fibras Por outro lado, grandes variaçðes na maturidade acabam por piorar a resistência da mecha, provavelmente pela aualidade inferior do próprio material celulósico das fibras. Conseqüentemente, várias tentativas de associar os índices "Pressley" com maturidade conduziram a baixos 'valores de correlação, freqüentemente negativos (PATEL \& PATIL, 1975, e SABINO et alii 1976), contrariamente ao que seria esperado pelas conclusões de MOORE (1941), isto é, uma correlação significativa, positiva.

Considerando que a maturidade é uma questão de grossura relativa das paredes da fibra e que o fibrógrafo é um aparelho de precisão que mede indiretamente esse parâmetro, através da densidade ótica de uma amostra de fibras paralelas (GRIDI-PAPP \& SABINO, 1972), o propósito do presente trabalho foi examinar a possibilidade de corrigir, para uma mesma amostra, as leituras de índice Pressley por meio de dados de densidade ótica obtidos no fibrógrafo. 


\section{MATERIAL E MÉTODOS}

\section{Considerações preliminares}

Sabe-se que as fibrilas que compõem as camadas celulósicas de uma fibra de algodão se dispðem em feixes que formam, com o eixo da fibra, certo ângulo, tanto menor quanto mais interna a camada examinada Consequentemente, quando a fibra é submetida à tração de uma força crescente, a seção que efetivamente resiste ao arrebentamento é ligeiramente menor que a seção total da sua parede (NEELAKANTAN, 1977). Por outro lado, para a mecha utilizada no aparelho "Pressley", o peso é proporcional à seção total das paredes das fibras, cujo comprimento é igualado (PRESSLEY, 1942). Menor grau de maturidade significa menor seçăo total das paredes, que é compensada pelo maior número de fibras, num mesmo peso de mecha. Essa compensação não acontece da mesma maneira com respeito à seção efetiva acima mencionada, e, no caso de pequenas variações da maturidade, resulta em uma tendência para maior resistência da mecha, em razão do maior número de fibras. Entretanto, essa tendência se inverte quando a porcentagem de fibras imaturas cresce muito, havendo uma acentuada diminuição das paredes das fibras e mudanças no próprio material celulósico. Essa ambigüidade das leituras de resistência diminui a confiabilidade dos dados em trabalhos de melhoramento, onde se almeja conhecer a resistência intrínseca das fibras e melhorá-la geneticamente. Torna-se necessária uma correção das leituras no sentido de expressar a resistência em termos de valor médio por fibra, em vez de valor relativo a um mesmo peso de mecha Tal correção se pode basear, em princípio, no conhecimento da espessura média das paredes das fibras que constituem a mecha levada ao aparelho.

Base teórica do processo proposto: Para determinada amostra de fibras, desde que sua quantidade no pente do fibrógrafo se localize dentro do intervalo recomendado para determinações de maturidade e de comprimento, a leitura inicial (L) do fibrógrafo ("Amount reading") é aproximadamente proporcional à espessura média das paredes das fibras $(\overline{\mathrm{d}}-\overline{\mathrm{do}})$ e ao número de fibras no pente $\left(n_{1}\right)$ :

$$
L=K_{1}(\bar{d}-\bar{d} 0) n_{1}
$$

onde $\mathrm{K}_{1}$ é uma constante de proporcionalidade (GRIDI-PAPP \& SABINO, 1972).

Sendo $P$ a leitura da resistência da mecha de $n_{2}$ fibras no aparelho Pressley e, RI, a resistência intrínseca das fibras, a resistência média da fibra individual pode ser expressa da seguinte forma: 


$$
\frac{P}{n_{2}}=K_{2}(\bar{d}-\overline{d o}) R I
$$

onde $\mathrm{K}_{2}$ é uma constante de proporcionalidade.

Dividindo-se a equação (2) por (1):

$$
\frac{P}{n_{2} L}=\frac{K_{2}}{K_{1}} \cdot \frac{R I}{n_{1}}
$$

ou

$$
R I=\frac{K_{1}}{K_{2}} \cdot \frac{P}{L} \cdot \frac{n_{1}}{n_{2}}
$$

Admitindo-se uma constante $K=K_{1} / K_{2}$ e considerando que, para uma mesma amostra homogeneizada, o peso médio de uma fibra é proporcional ao seu comprimento, ou seja:

$$
\frac{\mathrm{p}_{1} / \mathrm{n}_{1}}{1_{1}}=\frac{\mathrm{p}_{2} / \mathrm{n}_{2}}{1_{2}}
$$

onde $\mathrm{p}_{1}$ e $\mathrm{p}_{2}$ são, respectivamente, o peso de fibras do pente do fibrógrafo e o peso da mecha do Pressley, e $1_{1}$ e $1_{2}$ são os comprimentos das fibras no pente e na mecha, a relação (3) toma a seguinte forma:

$$
\begin{aligned}
\mathrm{RI} & =\mathrm{K} \frac{\mathrm{P}}{\mathrm{L}} \cdot \frac{\mathrm{p}_{1}}{\mathrm{p}_{2}} \cdot \frac{1_{2}}{1_{1}} \\
\text { ou } \quad \mathrm{RI} & =\mathrm{K} \frac{\mathrm{P}}{\mathrm{p}_{2}} \cdot \frac{\mathrm{p}_{1}}{\mathrm{~L}} \cdot \frac{1_{2}}{1_{1}} .
\end{aligned}
$$

O comprimento $1_{2}$ é de $15 \mathrm{~mm}$ (AMERICAN SOCIETY..., 1982b). Para $1_{1}$, considerou-se aceitável, como aproximação mais prática, o valor $2,5 \%$ do fibrógrafo, tomado em milímetros.

Conhecendo-se K, RI é calculada, para determinada amostra, obtendo-se os valores de $\mathrm{P}$ e $\mathrm{p}_{2}$ no Pressley e $\mathrm{L}, \mathrm{L}_{2,5} \%$ e $\mathrm{p}_{1}$ no fibrógrafo. 


\section{Material e Métodos}

As determinações foram realizadas em dois fibrógrafos Modelo 430 equipados com voltimetro e balança "Instant weight", visando a medidas de maturidade, aparelhos de Pressley $1 / 8$ " com balanças de torção e um aparelho Micronaire.

Utilizaram-se as amostras de fibras dos ensaios regionais de variedades conduzidos pela equipe de algodão do Instituto Agronômico, em 1981/ 82 , em 22 locais diferentes. Os estudos cobriram, desse modo, ampla faixa de variação em vista do ambiente e das diferenças genéticas existentes entre as seis variedades testadas nos ensaios, a saber: 'IAC 19', 'IAC 18', IAC 17-727 (lançada como 'IAC 20'), 'IAC 12-2', IAC 74/518 e 'IAC 17'. O delineamento dos ensaios foi em quadrado latino $6 \times 6$.

Realizaram-se estudos de regressão e correlação simples entre resistência intrínseca e índice Pressley e de cada um deles com indice Micronaire e maturidade, assim como de regressão múltipla e correlações parciais dos dois índices de resistência em função do comprimento " $2,5 \%$ ", uniformidade de comprimento, indice Micronaire e maturidade. A maturidade foi expressa em porcentagem de fibras maduras obtida pelo método do fibrógrafo (SABINO et alii, 1980). As demais determinações seguiram os processos padronizados ASTM (AMERICAN SOCIETY. . , 1982a, 1982b).

Foram comparadas, também, as análises de variância obtidas com dados de resistência intrinseca e de índices Pressley $1 / 8^{\prime \prime}$. Na comparação de médias, adotou-se o teste de Duncan, ao nivel de $5 \%$.

\section{RESULTADOS E DISCUSSÃO}

\section{Determinação da constante $K$}

Para conveniência dos trabalhos e visando obter valores de resistência intrínseca em grama/Tex, da mesma ordem de grandeza dos índices Presley $1 / 8$ ", determinou-se a constante $\mathrm{K}$, de tal maneira que, para o algodão padrão STF IAC 18 , fardo no 279 , de 1979 , o valor numérico de RI seja igual ao índice Pressley $1 / 8$ ". Para esse padrão, a equação (4) toma a seguinte forma:

$$
\begin{aligned}
\mathrm{RI} & =\frac{\mathrm{P}}{\mathrm{p}_{2}}, \log \mathrm{K}=\frac{\mathrm{L}}{\mathrm{p}_{1}} \cdot \frac{1_{1}}{1_{2}} \\
\text { ou } \quad \mathrm{K} & =\frac{1}{15} \cdot \frac{\mathrm{L}}{\mathrm{p}_{1}} \cdot \mathrm{L}_{2,5 \%}
\end{aligned}
$$


Os resultados de 1.400 determinações feitas nos dois fibrógrafos com o algodão padrão deram:

$$
\bar{K}=0,11383 \pm 0,00020
$$

com $s=0,00738$ e $\mathrm{CV}=6,48 \%$.

Com essas convenções, a expressão da resistência intrínseca, dada pela equação (4), toma, para qualquer material, a seguinte forma:

$$
\mathrm{RI}=15 \cdot 0,11383 \cdot \frac{\mathrm{p}}{\mathrm{p}_{2}} \cdot \frac{\mathrm{p}_{1}}{\mathrm{~L} \cdot \mathrm{L}_{2,5}}
$$

A unidade correspondente é grama/Tex.

\section{Correlações simples entre resistência, Micronaire e maturidade}

No quadro 1 são apresentados os coeficientes de correlação simples entre essas características Nota-se que há uma correlação relativamente alta entre resistência intrínsecae índice Pressley $1 / 8^{\prime \prime}, \mathrm{r}=0,626^{* *}$, apesar do problema apontado, da influência da maturidede. Quanto às correlações com Micronaire e maturidade, para o indice Pressley $1 / 8$ " não foram encontrados coeficientes significativos, enquanto a resistência intrínseca foi altamente correlacionada com ambas as variáveis

Merece destaque especial a correlação média para todas as variedades, com $r=0,695^{* *}$, entre a resistência intrínseca e a maturidade, mostrando a grande influência desta característica na resistência das fibras, o que seria de esperar mesmo intuitivamente. MOORE (1941), estudando, entre outros caracteres, a resistência média da fibra individual e a porcentagem de fibras de paredes finas em determinada amostra, verificou uma correlação média entre estas características de $r=0,688^{* *}$, com 58 graus de liberdade, bastante próxima do valor acima.

QUADRO 1 - Coeficientes de correlą̧ão simples entre resistência intrínseca (RI) e indice Pressley $1 / 8^{\prime \prime}(\mathrm{Pr})$, e de ambos com

\begin{tabular}{|c|c|c|c|c|c|c|c|}
\hline \multirow[b]{2}{*}{ Correlaçđ̃o } & \multirow[b]{2}{*}{$\mathrm{IAC} 19$} & \multirow[b]{2}{*}{ IAC 18} & \multicolumn{2}{|c|}{ Valores de I } & \multicolumn{2}{|l|}{$\ldots$} & \multirow{2}{*}{$\begin{array}{l}\text { Yalos } \\
\text { médio }\end{array}$} \\
\hline & & & IAC 17.727 & $1 \mathrm{AC} 12-2$ & IAC $74 / 518$ & $\mathrm{IAC} 17$ & \\
\hline$R J \times P J$ & $0,660^{* *}$ & $0,678 * *$ & $0,646^{* *}$ & $0,547^{* *}$ & $0,619 * *$ & $0,603^{* *}$ & $0,626^{* *}$ \\
\hline $\operatorname{Pr} \times M_{1}$ & 0,073 & 0,048 & 0,006 & 0,053 & $-0,009$ & 0,094 & 0,044 \\
\hline$R I \times M_{1}$ & $0,347^{* *}$ & $0,318^{* *}$ & $0,289 * *$ & $0,326^{* *}$ & $0,208 *$ & $0,404^{* *}$ & $0,315^{* *}$ \\
\hline $\operatorname{Pr} \times M \%$ & 0,166 & 0.157 & 0,086 & $-0,020$ & 0,070 & 0,048 & 0.085 \\
\hline $\mathrm{R} \mathbf{I} \times \mathbf{M} \%$ & $0,722 * *$ & $0,674^{* *}$ & $0,689 * *$ & $0,650^{* *}$ & $0,698^{* *}$ & $0,739 * *$ & $0,695 * *$ \\
\hline
\end{tabular}
indice Micronaire (Mi) e maturidade (Fıbrógrafo 430) (M\%)(')

(i) Todos os valores r foram estumados com 132 pares de dados. * Valor significativo ao nivel de 5\%.* Valor significativo ao nivel de $1 \%$. 
Com respeito a discrepâncias entre variedades, não houve diferenças dignas de consideração na maioria dos casos. Todavia, pelas correlações entre Pressley $1 / 8$ " e maturidade, nota-se um comportamento diferente para a 'IAC 19' e a 'IAC 18', com valores próximos do nível de significância.

\section{Correlações múltiplas}

As características comprimento $2,5 \%$, uniformidade de comprimento, Micronaire e maturidade foram consideradas as variáveis independentes e predeterminantes da resistência final das fibras. Por não constituir objeto de estudos, não se levou em consideração a conhecida influência da maturidade nas determinações do índice Micronaire. Em conseqüência, a participação da finura da fibra, expressa pelo índice Micronaire, foi superestimada.

As equações de regressão múltipla estão apresentadas no quadro 2 , e os coeficientes de correlação parcial, o de determinação e o valor " $F$ " do teste da regressão, no quadro 3 .

QUADRO 2 - Equaçōes de regressão múltipla do índice Pressley $1 / 8^{\prime \prime}(\operatorname{Pr})$ e da resistência intrínseca (RI), como variáveis dependentes, em função das variáveis comprimento da fibra 2,5\% (C), uniformidade de comprimento (U), índice Micronaire (Mi) e maturidade-fibrógrafo (M\%), obtidas para seis variedades de algodoeiro testadas em 22 ensaios regionais com seis repetiçōes $(\mathrm{N}=132)$

Variedade Equações de regressão para $\mathrm{Pr}$

IAC $19 \quad \mathrm{P}=-1,0008+0,3834 \cdot \mathrm{C}+0,3058 \cdot \mathrm{U}+0,0594 \cdot \mathrm{Mi}-0,0197 \cdot \mathrm{M} \%$

IAC $18 \quad \mathrm{P}=6,3133+0,1604 \cdot \mathrm{C}+0,2753 \cdot \mathrm{U}-0,1848 \cdot \mathrm{Mi}-0,0014 \cdot \mathrm{M} \%$

IAC 17.727 $\mathrm{P}=0,4530+0,3655 \cdot \mathrm{C}+0,2875 \cdot \mathrm{U}-0,0819 \cdot \mathrm{Mi}-0,0259 \cdot \mathrm{M} \%$

IAC 12-2 $\mathrm{P}=0,7442+0,3544 \cdot \mathrm{C}+0,2807 \cdot \mathrm{U}+0,2212 \cdot \mathrm{Mi}-0,0344 \cdot \mathrm{M} \%$

IAC 74/518 $\quad \mathrm{P}=10,8071+0,2545 \cdot \mathrm{C}+0,0983 \cdot \mathrm{U}-0,0009 \cdot \mathrm{Mi}+0,0021 \cdot \mathrm{M} \%$

IAC $17 \quad \mathrm{P}=3,6507+0,3705 \cdot \mathrm{C}+0,1567 \cdot \mathrm{U}+0,2954 \cdot \mathrm{Mi}-0,0135 \cdot \mathrm{M} \%$

Equações de regressão para $\mathrm{RI}$

IAC $19 \quad \mathrm{RI}=5,0798-0,3816 \cdot \mathrm{C}+0,3381 \cdot \mathrm{U}+0,0236 \cdot \mathrm{Mi}+0,2069 \cdot \mathrm{M} \%$

IAC $18 \quad \mathrm{RI}=12,6130-0,6106 \cdot \mathrm{C}+0,2897 \cdot \mathrm{U}-0,1512 \cdot \mathrm{Mi}+0,2336 \cdot \mathrm{M} \%$

IAC $17.727 \quad \mathrm{RI}=6,6646-0,3939 \cdot \mathrm{C}+0,3065 \cdot \mathrm{U}-0,0200 \cdot \mathrm{Mi}+0,1987 \cdot \mathrm{M} \%$

IAC 12-2 $\mathrm{RI}=7,3783-0,4252 \cdot \mathrm{C}+0,2947 \cdot \mathrm{U}+0,2722 \cdot \mathrm{Mi}+0,1980 \cdot \mathrm{M} \%$

IAC 74/518 $\mathrm{RI}=16,8777-0,5119 \cdot \mathrm{C}+0,1148 \cdot \mathrm{U}+0,0067 \cdot \mathrm{Mi}+0,2396 \cdot \mathrm{M} \%$

IAC $17 \quad \mathrm{RI}=10,1799-0,3746 \cdot \mathrm{C}+0,1784 \cdot \mathrm{U}+0,3166 \cdot \mathrm{Mi}+0,2000 \cdot \mathrm{M} \%$ 
QUADRO 3 - Coeficientes de correlação parcial, de determinação e teste F (variância regressão/variância residual) obtidos no estudo de correlação múltipla do índice Pressley 1/8" (Pr) e resistência intrínseca (RI), como variáveis dependentes, em função de comprimento de fibra 2,5\% (C), uniformidade de comprimento (U), índice Micronaire (Mi) e maturidade-fibrógrafo $(\mathrm{M} \%)$

\begin{tabular}{|c|c|c|c|c|c|c|}
\hline \multirow{2}{*}{ Variedade } & \multicolumn{4}{|c|}{ Coef. correlação parcial I } & \multirow{2}{*}{$\mathbf{R}^{2}$} & \multirow{2}{*}{$\mathrm{F}$} \\
\hline & Pr $\times \mathrm{C}$ & $\operatorname{Pr} \times \mathrm{U}$ & $\operatorname{Pr} \times \mathrm{Mi}$ & Pr $\times \mathrm{M} \%$. & & \\
\hline IAC 19 & $0,332 * *$ & $0,376^{* *}$ & 0,020 & $-0,073$ & 0,2540 & $10,81 * *$ \\
\hline IAC 18 & 0,158 & $0,372 * *$ & $-0,064$ & $-0,006$ & 0,1638 & $6,22 * *$ \\
\hline IAC $17-727$ & $0,289 * *$ & $0,341 * *$ & $-0,023$ & $-0,104$ & 0,1677 & $6,40^{* *}$ \\
\hline IAC $12-2$ & $0,345 * *$ & $0,349 * *$ & 0,071 & $-0,163$ & 0,2147 & $8,68 * *$ \\
\hline IAC $74 / 518$ & $0,212^{*}$ & 0,120 & $-0,003$ & 0,009 & 0,0681 & 2,32 . \\
\hline IAC 17 & 0,263 & $0,193^{*}$ & 0,083 & $-0,060$ & 0,1104 & $3,94 * *$ \\
\hline
\end{tabular}

Coef. correlação parcial $\mathbf{r}$

\begin{tabular}{|c|c|c|c|c|c|c|}
\hline \multirow{2}{*}{ Variedade } & & \multirow[b]{2}{*}{$\mathrm{R}^{2}$} & \multirow[b]{2}{*}{$\mathrm{F}$} \\
\hline & $\mathrm{RI} \times \mathrm{C}$ & $\mathrm{RI} \times \mathrm{U}$ & $\mathrm{RI} \times \mathrm{Mi}$ & $\mathrm{RI} \times \mathrm{M} \%$ & & \\
\hline IAC 19 & $-0,342 * *$ & $0,422 * *$ & 0,008 & $0,625 * *$ & 0,6480 & $58,44^{* *}$ \\
\hline IAC 18 & $-0,521 * *$ & $0,390 * *$ & $-0,053$ & $0,717^{* *}$ & 0,6781 & $66,89 * *$ \\
\hline IAC $17-727$ & $-0,325^{* *}$ & $0,379 * *$ & $-0,006$ & $0,646^{* *}$ & 0,6131 & $50,31 * *$ \\
\hline IAC $12-2$ & $-0,398 * *$ & $0,360 * *$ & 0,086 & $0,682 * *$ & 0,5880 & $45,32 * *$ \\
\hline IAC $74 / 518$ & $-0,400 * *$ & 0,140 & 0,002 & $0,706 * *$ & 0,5766 & $43,24 * *$ \\
\hline IAC 17 & $-0,284^{* *}$ & $0,234^{*}$ & 0,096 & $0,694 * *$ & 0,6189 & $51,57 * *$ \\
\hline
\end{tabular}

* Significativo ao nível de $5 \%$. ${ }^{* *}$ Significativo ao nível de $1 \%$.

Tanto os coeficientes de regressão como os de correlação parcial mostram que as correlações referentes à resistência intrínseca são mais consistentes do que as relativas ao indice Pressley 1/8'. Por outro lado, enquanto não explicou quase nada da variação deste último, a maturidade foi um dos principais fatores na variação da resistência intrínseca $\mathrm{O}$ coeficiente de determinação variou no caso do Pressley $1 / 8$ " em torno de 0,163 e, para a resistência intrínseca, em torno de 0,620 , enquanto para $F$ obtiveram-se valores em torno de 6,4 e 52,6 respectivamente Em ambos os casos de regressão múltipla, não houve influência do indice Micronaire, e a uniformidade de fibra influiu tanto quanto o comprimento $2,5 \%$. Com respeito à uniformidade, 
houve diferença sensivel entre materiais, distinguindo-se das demais a variedade ' $\mathrm{IAC} 17$ ' e a linhagem IAC 74/518. A resistência intrínseca correlacionou-se negativamente com comprimento $2,5 \%$, na ausência de variação genética.

\section{Análise dos ensaios}

No quadro 4 são apresentadas as médias de índice Pressley $1 / 8$ " e de resistência intrínseca obtidas nos 22 ensaios regionais, para cada variedade estudada, assim como os respectivos valores de $F$ para tratamento.

Verifica-se que houve modificação na classificação dos tratamentos quando se passou do indice Pressley $1 / 8$ " para a resistência intrínseca. A 'IAC 18' e a 'IAC 12-2', intermediárias, no primeiro caso, melhoraram de posição, ocorrendo o inverso com a 'IAC 19'. Aparentemente, o problema da interferência da maturidade nas leituras de Pressley 1/8" foi maior para as duas primeiras e menor para a última. De maneira geral, houve maior discriminação entre variedades no caso da resistência intrínseca.

QUADRO 4 - Médias dos tratamentos e resultados das análises estatísticas conjuntas para indice Pressley $1 / 8$ " $(\mathrm{Pr})$ e para resistência intrínseca (RI) obtidos em 22 ensaios regionais de variedades de algodoeiro

\begin{tabular}{llc}
\hline \multirow{2}{*}{ Variedade } & \multicolumn{2}{c}{ Médias $\left({ }^{1}\right)$} \\
\cline { 2 - 3 } & \multicolumn{1}{c}{ Pr } & RI \\
\hline IAC 19 & $22,30 \mathrm{a}$ & $20,92 \mathrm{~b}$ \\
IAC 18 & $22,03 \mathrm{bc}$ & $21,63 \mathrm{a}$ \\
IAC 17-727 & $21,68 \mathrm{~d}$ & $20,51 \mathrm{c}$ \\
IAC $12-2$ & $21,85 \mathrm{~cd}$ & $21,02 \mathrm{~b}$ \\
IAC 74/518 & $22,21 \mathrm{ab}$ & $21,42 \mathrm{a}$ \\
IAC 17 & $20,93 \mathrm{e}$ & $19,64 \mathrm{~d}$ \\
\hline F & 41,05 & 40,25 \\
CV \% & 3,1 & 5,0 \\
\hline
\end{tabular}

( ${ }^{1}$ ) Médias seguidas da mesma letra não diferem entre si pelo teste de Duncan a 5\%. 


\section{CONCLUSÕES}

Os resultados apresentados permitem formular as seguintes conclusões:

1. A resistência intrinseca da fibra de algodão pode ser expressa pela fórmula: $R I=15 \cdot 0,11383\left(P / p_{2}\right)\left(p_{1} / L . L_{2,5}\right)$, onde $P$ é o peso de arrebentamento de uma mecha de fibra, de uma dada amostra homogênea, no aparelho Pressley $1 / 8 ", \mathrm{p}_{2}$ é o peso da mecha, L é a leitura inicial, no fibrógrafo, do material de um pente da mesma amostra, $p_{1}$ o peso desse material e $L_{2,5 \%}$ a leitura do comprimento $2,5 \%$.

2. A resistência intrínseca, assim definida, apresentou alta correlação $\left(\mathrm{r}=0,626^{* *}\right)$ com o índice Pressley $1 / 8^{\prime \prime}$.

3. Na ausência de variações genéticas, a resistència intrínseca apresentou alta correlação com maturidade $(\tau=0,695)$, como era de esperar, enquanto o índice Pressley $1 / 8$ " não se correlacionou com essa característica $(\mathrm{r}=0,085)$.

4. Admitindo-se, na ausência de variações genéticas, a resistência média da fibra como dependente do comprimento, da uniformidade de comprimento, do índice Micronaire e da maturidade, no caso do índice Pressley $1 / 8$ ", verificou-se contribuição significativa somente dos dois primeiros fatores, enquanto, para a resistência intrínseca, a maior influência foi da maturidade, vindo, em seguida, a uniformidade de comprimento e o comprimento 2,5\%. Esta se correlacionou negativamente com a resistencia intrínseca.

5. Na análise conjunta de ensaios regionais, a classificação das variedades mudou quando se considerou a resistencia intrínseca em vez do índice Pressley $1 / 8$ ", havendo maior discriminação entre variedades.

6. A expressão proposta de resistência intrínseca mede melhor a resistência média de fibras individuais do que 'o índice Pressley $1 / 8$ " e poderá ser utilizada com vantagem pelos melhoristas e, possivelmente também, pelos usuários da fibra de algodãa

\section{SUMMARY}

\section{INTRINSIC FIBER STRENGHT OF COTTON ESTIMATED THROUGH CORRECTION OF THE PRESSLEY INDEX}

The possibility to estimate the mean fiber strength by correcting Pressley 1/8" determinations through Fibrograph measures is demonstrated. The intrinsic strength (RI) of fibers of a homogenized sample may be obtained by the following expression: $\mathrm{RI}=15 \times 0.11383\left(\mathrm{P} / \mathrm{p}_{2}\right)\left(\mathrm{p}_{1} / \mathrm{L} \times \mathrm{L}_{2.5}\right.$ f $\left._{0}\right)$ where $\mathrm{P}$ is the Pressiey reading, $\mathrm{P}_{2}$ is the weight of the bundle of nibers, $L, L_{2.5} \%$ and $\mathrm{p}$, are the amount reading, the $2.5 \%$ staple length and the weight of fibers on the comb of the Fibrograph. Compara- 


\begin{abstract}
tive studies carried out by the authors in the Laboratory of Fiber Technology of the "Instituto Agronomico" at Campinas, State of São Paulo, Brazil, about the two measures of fiber strenght showed that, whereas the Pressley $1 / 8$ " indices had no correlation with Micronaire and maturity, in the abscence of genetic variations, the RI values where highly correlated with both characters. Among the main fiber characters, maturity had the highest contribution to variations of $\mathrm{RI}$ in multipie regression analysis. The stidy of 22 regional variety testes showed that data of intrinsic srength yielded higher discrimination among varieties than those of Pressley $1 / 8$ "
\end{abstract}

Index terms: cotton; intrinsic resistance; corrected Pressley.

\title{
REFERENCIAS BIBLIOGRAFICAS
}

AMERICAN SOCIETY FOR TESTING AND MATERIALS, Philadelphia. Annual Book of ASTM Standards, Part 32. Philadelphia, 1982a. $1136 \mathrm{p}$. $926 \mathrm{p}$. Annual Book of ASTM Standards, Part 33. Philadelphia, 1982b.

BURLEY JR, ST. \& CARPENTER, F. The evaluation of results obtained on available types of fiber strength testers using various gauge spacings and their relation to yarn strength. Washington, U.S. Dep. of Agriculture, Agricultural Marketing Service, 1955. 19p. (AMS, 71)

CHANDLER, EE A new method for determining the strength of cotton. A preliminary report. Washington D.C., U.S. Dep. of Agriculture and Clemson Agricultural College, 1926. 16p.

DE COENE, R. \& VERSCHRAEGE, L.M. Contribution à l'étude des facteurs influençant la resistance des fibres de coton, mesurée and dynamomètre de Pressley. Annales Tex tiles, 4:7-48, 1963.

DELUCA, L.B. The intrinsic tenacities of natural cellulosic fibers Textile Research Journal, 34:110-116, 1964.

GRIDI-PAPP, I. L \& SABINO, N.P. Maturidade da fibra de algodão determinada por processo ótico - Método do Fibrógrafo Digital. Revista Brasileira de Tecnologia, São Paulo, 3:99-106, 1972.

MOORE, J.H. The distribution and relation of fiber population length, breaking load, weight, diameter and percentage of thin-walled fibers on the cotton seed in five varieties of american Upland cotton Journal of Agricultural Research, 62:255-302, 1941.

NEELAKANTAN, P. The ideal cotton-fibre strength and its relation to observed strength, maturity and fineness. Textile Research Journai, 47 . $178-181,1977$. 
PATEL, G.S \& FATIL, N.B. Studies on some physical parameters of cotton fibers and their influence on breaking strength Textile Research Journal, 45:168-172, 1975.

PRESSLEY, EH A cotton fiber strength tester. ASTM Bulletin, October: $13-17,1942$

SABINO, N.P.; GRIDI-PAPP, IL \& GROSSI, J.M.M. Correlações entre a maturidade da fibra e outros caracteres economicos do algodoeira Bragantia, Campinas, 35:375-379, 1976. ; ; KONDO, J.I. \& CARNEIRO, J.B. Maturidade da fibra de algodão determinada pelo Fibrógrafo Modelo 430. Bragantia, Campinas, 39:69-77, 1980. 